\title{
Mobile-to-clogging transition in a Fermi-like model of counterflowing particles
}

\author{
Roberto da Silva and Eduardo V. Stock \\ Institute of Physics, Federal University of Rio Grande do Sul, Porto Alegre-RS, 91501-970, Brazil
}

(Received 1 September 2018; published 29 April 2019)

\begin{abstract}
In this paper we propose a generalized model for the motion of a two-species self-driven objects ranging from a scenario of a completely random environment of particles of negligible excluded volume to a more deterministic regime of rigid objects in an environment. Each cell of the system has a maximum occupation level called $\sigma_{\max }$. Both species move in opposite directions. The probability of any given particle to move to a neighboring cell depends on the occupation of this cell according to a Fermi-Dirac-like distribution, considering a parameter $\alpha$ that controls the system randomness. We show that for a certain $\alpha=\alpha_{c}$ the system abruptly transits from a mobile scenario to a clogged state, which is characterized by condensates. We numerically describe the details of this transition by coupled partial differential equations (PDE) and Monte Carlo (MC) simulations that are in good agreement.
\end{abstract}

DOI: 10.1103/PhysRevE.99.042148

\section{INTRODUCTION}

The theoretical motion of particles in inhomogeneous media with local impurities can be observed in many contexts in Physics and in a large number of applications such as capture and decapture of electrons in micro, nano, and meso devices [1-3], the erratic, but also directed, motion of molecules in chromatographic columns [4], and many others. However, other situations that consider particles interaction can be explored, and we will particularly focus on the one that takes into account two different species of particles moving against each other.

The patterns arising from counterflowing stream of particles can be studied considering a wide range of apparently very different systems such as pedestrian dynamics [5], and charged colloids motion [6,7], which suggest more similarities between the micro and macro systems that we can anticipate in this kind of modeling. For this reason the straight formation of lanes, distillation, originated from the complex emergent process of self-propelled and/or field-directed objects and particles have raised a lot of interesting questions in the context of statistical mechanics and the physics of stochastic process modelled by Monte Carlo (MC) simulations or partial differential equations (PDE) [8,9].

Similarly, more fundamental situations related to systems that collapse due to clogging effects, related to the typical phenomena of concentration of objects, or their condensation patterns, under counterflowing streams, lead to a fundamental question: How the environmental randomness compares to the size of objects for the occurrence of clogging and jamming phenomena? To understand this interesting problem that relates the micro with the macro scales, in this work we consider a general modeling of streams of counterflowing objects interpolating two very distinct situations:

(1) Situation (i): Objects of negligible sizes move in random environment and their excluded volume is not a relevant parameter. In this case the system is entirely random: the particle performs a biased random walk in which it makes a step to the next cell with probability $1 / 2$ or remains at the original cell with the same probability. It works as if the randomness of the motion is due to the resistance offered by the environment and not from the interaction among objects. This randomness is then not affected by the particle size.

(2) Situation (ii): The second extreme situation considers hard objects where the excluded volume plays a role. It works as if rigid bodies interact only with each other. In this situation the system is essentially deterministic and one object does not move to a cell that does not have enough space to allocate it.

In this work we explore the transition between these two distinct scenarios, by changing an external parameter denoted by $\alpha$. We are able to map continuously from situation (i) ( $\alpha=$ 0 ) to situation (ii) $(\alpha \rightarrow \infty)$ and the interesting point is how it is performed.

For that, we propose a simple model of two species of objects, denoted by $A$ and $B$, moving in opposite directions in a ring divided by cells, which have the same maximum occupation denoted by $\sigma_{\max }$ that depends on the stochasticity parameter $\alpha$. To describe $\alpha$, which controls in which degree the maximal occupation $\sigma_{\max }$ can be violated, we use an adapted Fermi-Dirac distribution that governs the particle transition between the cells.

It is worth to emphasize that our model can represent a lot of different systems, among them, for example, oppositely charged colloids in counterflowing streams. In this context, the application of a strong electric field along the longitudinal direction of the ring, would make species $A$ drift, let us say, in a counterclockwise fashion while species $B$ would drift in the opposite direction or we can picture objects entering and leaving both extremities of a thin tape (tube) at a constant rate (periodic boundary conditions), or as a last mention, the model proposed can also mimic typical situations of pedestrians walking in subway corridors under some peculiar conditions.

With our adapted Fermi-Dirac distribution we are able to describe the complex dynamics of particles clogging in counterflowing that here is studied in both: PDE and MC simulations. Our results indicate the existence of a transition from a mobile phase (fluid) to clogged (condensate) phase, 
by monitoring the evolution of the particle concentration patterns.

In Sec. II we present the details of the model. Our main results are presented in Sec. III. Finally we summarize our results in Sec. IV and we present our main conclusions.

\section{THE MODEL}

We consider in this paper a two-species model of particles which drift in counterflow in an annular system composed by $L$ cells, each one with the same limiting factor $\sigma_{\max }$, regarding the distinct situations (i) and (ii) described in the previous section. For the sake of simplicity, we pictorially illustrate our idea in Fig. 1.

Considering that the concentration of particles (of whatever species) in the following cell affects the locomotion of the particles in the current cell, the concentration of target objects, according to our prescription can be written by the recurrence relation: $A_{m, n}=p_{m-1, m}^{(n-1)} A_{m-1, n-1}+p_{m, m}^{(n-1)} A_{m, n-1}$, where $A_{j, k}$ is the density of particles of species $A$ of the cell $j$ at time $k$ and by construction $p_{m, m}^{(n-1)}+p_{m, m+1}^{(n-1)}=1$, since here $p_{i, j}^{(n)}$ denotes the probability of particle in cell $i$ (position $x=i \varepsilon$ ) to transit to cell $j$ (position $x=j \varepsilon$ ) at $t=n \tau$, where $\tau$ means the required time to perform such transition and $\varepsilon$ is the step length.

Combining the equations, one has the following equations:

$$
A_{m, n}-A_{m, n-1}=p_{m-1, m}^{(n-1)} A_{m-1, n-1}-p_{m, m+1}^{(n-1)} A_{m, n-1} .
$$

Taking into account that the occupation depends on a maximum level of occupation, it is interesting to use the analogy of the Fermi level in the context of conductor and semiconductor models, and that here it has a similar role changing the temperature by a factor $\alpha^{-1}$. If the desired cell has a number of objects above the maximum level, then the probability of occupation behaves according to Fermi-Dirac occupation function:

$$
p_{i, j}^{(n)}=\left(1+\exp \left[\alpha\left(\sigma_{j, n}-\sigma_{\max }\right)\right]\right)^{-1},
$$

where $\sigma_{j, n}=A_{j, n}+B_{j, n}$ denotes the total number of objects at the cell $j$, at the time $n$, which is the sum of the number

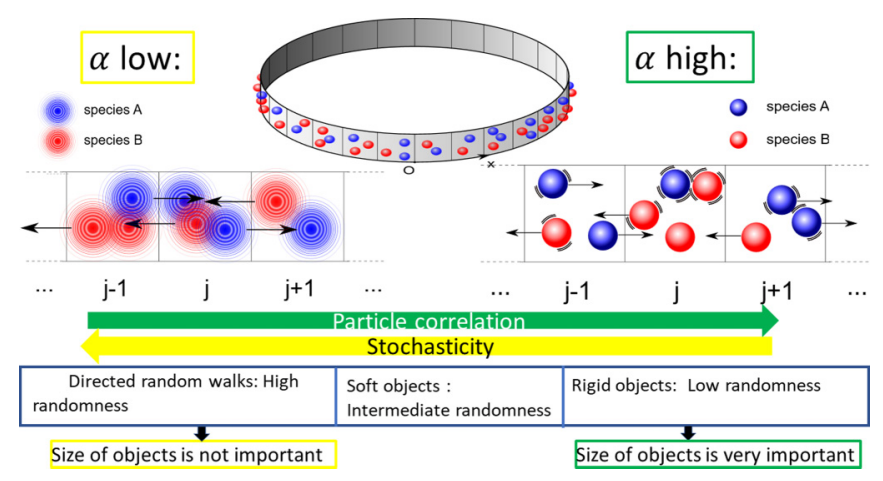

FIG. 1. Section of particles under counterflowing in a ring topology. The two extreme regimes are illustrated: $\alpha \rightarrow 0$, showing that objects are independent of each other but interact with the stochastic environment, and $\alpha \rightarrow \infty$ where interacting rigid bodies with high momenta ignore the randomness of the environment. Intermediate values of $\alpha$ correspond to some situation between these two extremities. of objects of target species and the number of the objects of opposite species $B_{j, n}$. The choice of Fermi-Dirac function to model the stochastic process of cell occupation is very natural at this point: If the concentration of arrival cell $A_{j, n}+B_{j, n}$ is greater than $\sigma_{\max }$, then the transition is hampered; otherwise, the transition is facilitated. How much is hampered or facilitated depends only on $\alpha$ which is not exactly the inverse of temperature, but the matching between the FermiDirac distribution and our desired mapping is surprisingly meaningful. The objects can occupy the cell even when $A_{j, n}+$ $B_{j, n}>\sigma_{\max }$. The only case this does not occur is when $\alpha \rightarrow \infty$, since $p_{i, j}^{(n)}=1$ if $\sigma_{j, n}=A_{j, n}+B_{j, n}<\sigma_{\max }, p_{i, j}^{(n)}=$ $1 / 2$ if $\sigma_{j, n}=\sigma_{\max }$, and $p_{i, j}^{(n)}=0$ when $\sigma_{j, n}>\sigma_{\max }$. This case (corresponding to situation (ii) previously considered) means that no more than $\sigma_{\max }+1$ objects per cell are allowed. We consider that hard core objects interact and the environment has no influence on their motion.

When $\alpha \rightarrow 0$, which corresponds to a low field regime, one has $p_{i, j}^{(n)}=1 / 2$ meaning that the objects do not interact with each other, only with environment. In this case (corresponding to situation (i)), we can imagine that an infinity number of objects (although not likely) are allowed per cell, since the motion does not depend on $\sigma_{\max }$. Since the interaction among the particles is not considered in this limit, the objects are drifting in the environment.

It is important to notice that our model prescribes other alternative interpretations. One of them can imagine $\alpha$ as a kind of field that drives the objects oppositely charged as considered, for example, in the interesting stochastic lattice gas studied by Schmittmann et al. [10]. In our modeling, we can imagine that for low $\alpha$, the environment is important since the momenta of objects are low. On the other hand when $\alpha$ is large, the objects have high momenta and the environment effects are not important to change the velocities of these objects. In this case the interaction of objects have an important role.

Thus, one has for the objects $A$, the recurrence relation $A_{m, n}=A_{m, n-1}+a_{m-1, n-1}-a_{m, n-1}$, and similarly for the objects $B$, the relation $B_{m, n}=B_{m, n-1}+b_{m+1, n-1}-b_{m, n-1}$ with $a_{m, n} \equiv A_{m, n} /\left[1+e^{\alpha\left(A_{m+1, n}+B_{m+1, n}-\sigma_{\max }\right)}\right]$ and $b_{m, n} \equiv B_{m, n} /[1+$ $\left.e^{\alpha\left(A_{m-1, n}+B_{m-1, n}-\sigma_{\max }\right)}\right]$.

One can solve the recurrence relation as we do in this paper, but we also analyze the corresponding differential equation. By considering the situation $A_{m+1, n}+B_{m+1, n} \approx A_{m-1, n}+$ $B_{m-1, n} \approx A_{m, n}+B_{m, n}$, we are led to a system of two coupled equations:

$$
\frac{\partial A(B)(x, t)}{\partial t}=-(+) C \frac{\partial}{\partial x}\left[\frac{A(B)(x, t)}{1+e^{\alpha(A(x, t)+B(x, t)-\sigma)}}\right],
$$

where $C=\lim _{\tau, \varepsilon \rightarrow 0} \frac{\varepsilon}{\tau}$. It is important to notice that when $\alpha \rightarrow 0$, we have uncoupled equations. In this situation, the solutions are expected to satisfy $\frac{\partial A(x, t)}{\partial t}=-C \frac{\partial A(x, t)}{\partial x}$ and $\frac{\partial B(x, t)}{\partial t}=C \frac{\partial B(x, t)}{\partial x}$. For $C=1$, for example, under periodic boundary conditions, $A(x=L, t)=A(x=0, t)$ and $B(x=$ $L, t)=B(x=0, t)$, it is easy to verify that the only possibility is $A(x, t)=1$ and $B(x, t)=1$, with the initial conditions $A(x, t=0)=1$ and $B(x, t=0)=1$.

In the discrete formulation, this means $p_{i, j}^{(n)}=1 / 2$, which corresponds to a ballistic behavior of objects, since if one 
considers $A(x, t=0)=L \delta_{x, 0}$ and $B(x, t=0)=L \delta_{x, L}$, we expect the solutions of recurrence relations, in the first round in the ring, to be given by $A_{m, n} \approx L\left(\begin{array}{c}n-1 \\ m-1\end{array}\right) 2^{-n}$ and $B_{m, n} \approx$ $L\left(\begin{array}{c}n-1 \\ L-m-1\end{array}\right) 2^{-n}$, once to execute $m$ success (it means to be in the position $x=m \tau)$ the particles have to execute $n>m$ experiments according to a negative binomial [11]. Here we are making the number of cells exactly the length of the tube (or simply $\varepsilon=\tau=1$ ).

However, after several rounds, we must expect that $A_{m, n}$, $B_{m, n} \rightarrow 1$. But what happens when $\alpha$ increases and the interactions between particles start to become important? We also expect that $A_{m, n}, B_{m, n} \rightarrow 1$, which means that particles are transiting without clogging in the channel?

To analyze this point we also consider performing Monte Carlo (MC) simulations to support the numerical integration of the recurrence equations. In these MC simulations, we consider synchronous updating (every particle is verified if it goes to the next cell or stays stopped at the same cell). In this case there is no possibility of the same particle to be tested. The initial configuration considered in such simulations supposes that all particles are randomly (uniformly) distributed among the cells.

Moreover, MC simulations make possible to analyze the clogging dynamics in such kind of systems by considering an interesting order parameter that measures a kind of current of objects over the annular tube, here called as mobility, which is defined for $N$ particles at time $t$ as

$$
M(t)=\frac{1}{N} \sum_{i=1}^{N} \xi_{i}(t),
$$

where $\xi_{i}(t)$ is a binary variable associated to particle $i$, which assumes 0 if the particle stays stopped at time $t$ and 1 if this same particle goes to the next cell at this same time. This quantity cannot be calculated by solution of recurrent relations, but when we perform MC simulations it is easily obtained since we work exactly on the particles, differently from recurrence relations. Some authors call this amount simply as current.

First, let us to better explore the variation of $\alpha$. We solve the recurrence relations starting with initial conditions $A_{m, 0}=$ 1 and $B_{m, 0}=1$, but with only one site $m=L / 2$, empty, i.e., $A_{L / 2,0}=B_{L / 2,0}=0$. Differently from MC simulations we need an initial defect to promote the time evolution of the system when we numerically integrate the recurrence relations. So we expect that for low $\alpha, A_{m, n} \rightarrow 1$ (in our particular case, $A_{m, n} \rightarrow \frac{(L-1)}{L} \approx 1$, for $L$ large). On the other hand, i.e., for higher values of $\alpha$, the question is: for which values of $\alpha$ the system breaks down in a clogging situation?

In next section we present the main results of this work.

\section{RESULTS}

Let us initially consider the simplest case $\sigma_{\max }=1$, and let us start by observing the density of particles $A$ and $B$ in both methods: MC simulations and by numerical integrations of recurrence relations (REC). A summary of our main results can be seen in Fig. 2. Figure 2(a) shows that for $\alpha=0.3$ the system is freely flowing since for averaging both species over

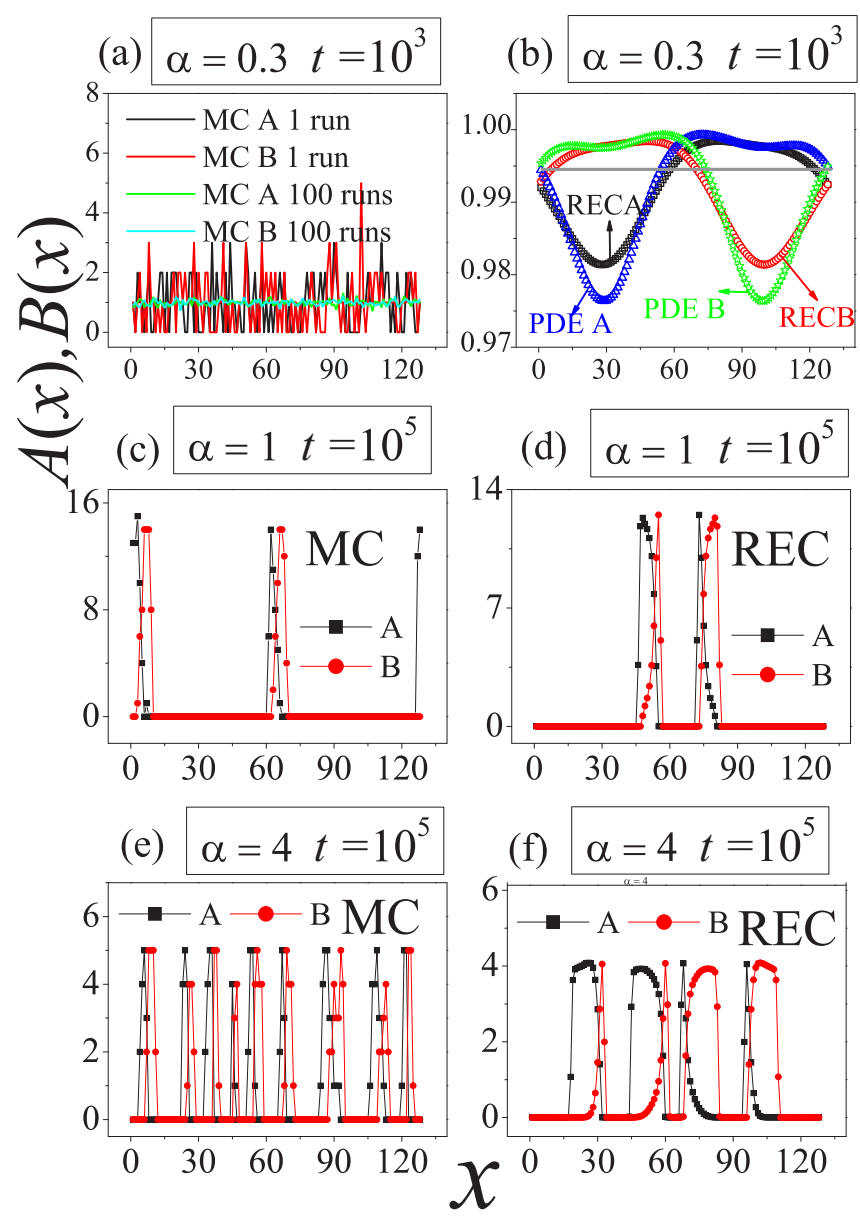

FIG. 2. Exploring the dynamics for different values of $\alpha$, methods (MC and REC), and number of time steps.

a large number of runs $\left(N_{\text {run }}=100\right)$ we have $A(x) \approx B(x) \approx$ 1. It is important to observe that for $N_{\text {run }}=1$ the fluctuations overcome the expected behavior.

The numerical solution was also obtained via two independent methods: numerical solution of PDE according to Eq. (1) and REC solutions, which simply integrate the previous recurrence relations used to deduce the PDE of the problem, for the same value of $\alpha$, which have no obligation to agree, but we expect that them to have at least the same qualitative behavior. The results are shown in Fig. 2(b). We can observe that although to obtain the PDE we have changed the index in the recurrence relation, the methods show curves around $A(x)=B(x)=1$ for intermediate time, $t=10^{3}$. Moreover, in the same plot, for $t=10^{5}$ steps, the straight gray line represents all plots obtained from PDE and REC solutions that are coincident, which indicates an exact agreement with $A(x)=B(x)=1$. From now on, we will use only REC in this paper, since PDE only presents some slightly differences in relation to the first one and it can be considered a good representation of the model via partial differential equations. Other mathematical properties of PDE in these counterflowing problems deserve future exploration.

How about when $\alpha$ increases? For example for $\alpha=1$, both $\mathrm{MC}$ and REC indicate some points of clogging characterized by high density of particles $14 \lesssim A \approx B \lesssim 16$ according to 

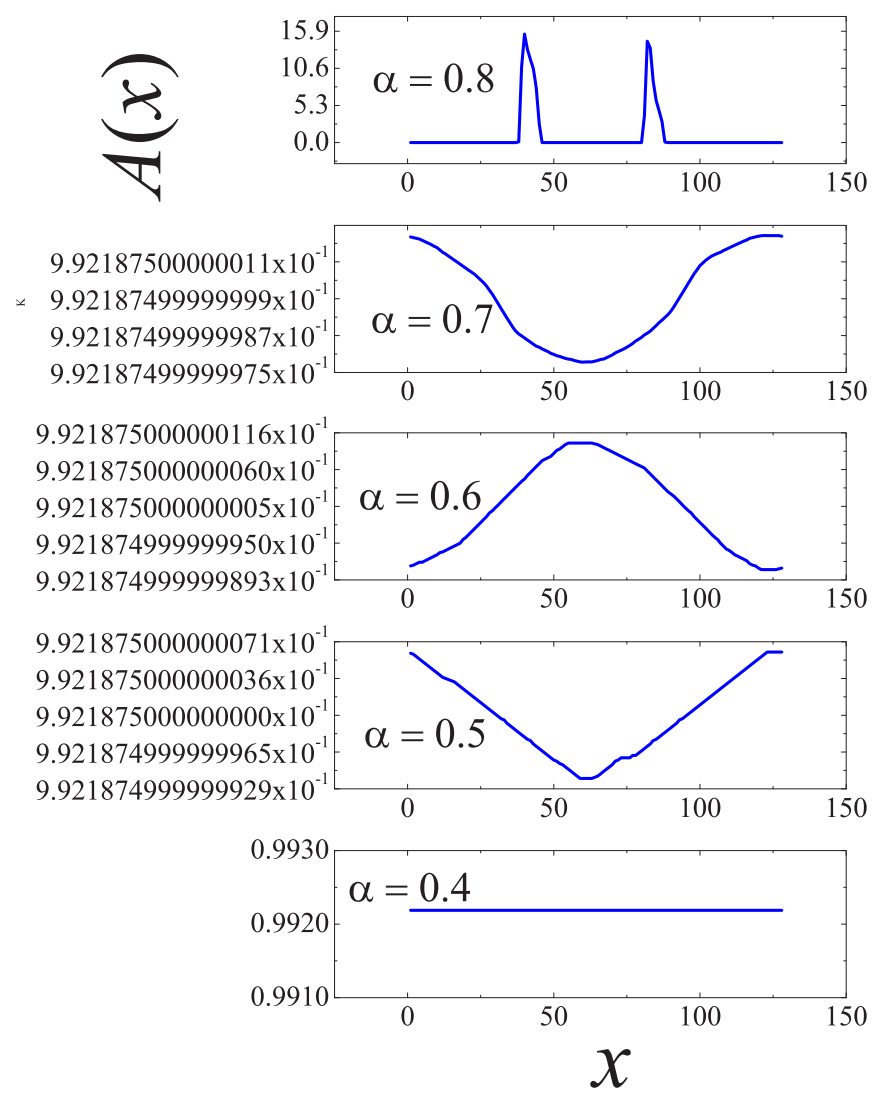

FIG. 3. Density of particles $A$ by REC solutions for $t=10^{5}$ iterations (steady state). Clearly the systems is mobile for $\alpha=0.4$ and jammed for $\alpha=0.8$ (formation of condensates). We can observe a "strange" behavior in the vicinity of transition $(0.5 \leqslant \alpha \leqslant 0.7)$.

Figs. 2(c) and 2(d) showing that both methods bring such situation. It is interesting, since when we have $\alpha=4$ the jamming occurs with many situations of "bottlenecks" but now it occurs with lower magnitude $3 \lesssim A \approx B \lesssim 4$ exactly as shown in Figs. 2(e) and 2(f). So we raise the question, about the existence of $0<\alpha_{c}<\infty$ for which the system transits from mobile to clogged situation in the case $\sigma_{\max }=1$.

Here it is important to mention that differently from other works our results look at transition dependence on the randomness of system $(\alpha)$. Other works do not consider parameter $\alpha$, and in pedestrian dynamics the authors work with the transition of some parameter as average system velocity or probability of clogging versus the density of pedestrian (see for example interesting works: respectively, Refs. [12] and [13]).

First, by considering the vicinity of transition, we look the density of $A$ for five different values of $\alpha$ considering the stationary situation $t=10^{5}$ iterations (see Fig. 3). For example we have a mobile system for $\alpha=0.4$ and a system completely jammed for $\alpha=0.8$ (two pronounced peaks) but for intermediate values of $\alpha(0.5,0.6$, and 0.7$)$ the REC solutions show that system seems to be in a metastable situation, where $A \approx 1$, but slightly numerical differences are able to deform the solution leading to strange shapes until to arrive the clogged situation $\alpha=0.8$ starting from the mobile situation $\alpha=0.4$.
Thus, it goes into action, the mobility defined by Eq. (2), and with this concept we can better understand what indeed is happening with the system. Here we look at time evolution of the mobility considering a large number of time steps $\left(t_{\max }=\right.$ $10^{9} \mathrm{MC}$ steps) in order of our stop criterion to fail (what does not happen). Basically the mobility arrives a steady state $M_{\infty}$ (stationary mobility). We use a criterion to analyze when the system reaches this stationary mobility. First, we observe the system visually, which corresponds to a qualitative previous analysis. Secondly, we take a slope of the stationary mobility by lags of $10^{3} \mathrm{MC}$ steps. When the slope is lesser than (in absolute value) $\eta$ we consider that system has reached the stationarity. We use $\eta=10^{-7}$, and we check these cases with our previous visual analysis. After these considerations, we analyze the behavior of $M(t)$ for fixed values of density as function of $\alpha$ taking the stationary value for each value.

We performed simulations for several values of systems $L$. Figures 4(a) and 4(b) show, respectively, the time evolution of mobility for $L=8$ and $L=256$. Here we average the mobility over considerable number of runs: $N_{\text {run }}=L^{-1} 10^{6}$ runs. We can observe that plots are really different. So it is interesting to check the stationary mobility for different size systems as function of $\alpha$ which is observed in Fig. 4(c). We can check that system is deeply sensitive on the size system, but for $L \geqslant 128$, no numerical differences were observed and Fig. 4(d) shows the results for $L=256$ considering different densities from $\rho=0.062$ up to 1 . Coming back to Fig. 4(c), it is important to mention an anomalous recovering of mobility for large values of $\alpha$, but such distortion only occurs for really small systems, which is not relevant.

These results show an abrupt transition between a mobile phase $\left(m_{\infty}>0\right)$ to a clogging phase $\left(m_{\infty}=0\right)$. This transition is preceded by an initial slip of mobility. This occurs because when the interaction of the environment with objects decreases, i.e., $\alpha$ enlarges, the objects gain an initial increase of the mobility, given their high momenta. But as $\alpha$ enlarges even more, the interaction among the objects really increases, until it finally destroys the mobility. In this case the motion is random only when cell occupation assumes exactly the value $\sigma_{\max }$. The high momenta of the objects that ignore the environment is an important constraint to make the system reaches the clogging situation due to the strong interaction effects among the objects. It is interesting to observe that the abrupt transition to a clogging phase occurs with a high peak of density immediately followed by a large number of smaller peaks of bottlenecks, as suggested by Figs. 2 and 3 .

This analysis concerned $\sigma_{\max }=1$. So the question is, should we observe anomalous effects for $\sigma_{\max }>1$, which in our systems means to consider small objects or simply, more "particles" occupying the same "orbital" ? In this case can we observe a clogging transition for a $\alpha_{c}^{(1)}$ and a recovering of mobility of the system for a $\alpha_{c}^{(2)}>\alpha_{c}^{(1)}$ ? Yes, it occurs. So we analyze simulations of mobility considering now $\sigma_{\max }>1$. In this case it is important to make a distinction, density and occupation. We define density by $\rho=\frac{N}{L}$ where $N$ is the number of particles and $L$ the system size, or simply the number of cells. Occupation is a different concept, which here is defined as $o=\frac{N}{\sigma_{\max } L}$. Thus, we prepared two experiments, so that in one of them, we change $\sigma_{\max }$ keeping the density constant, 

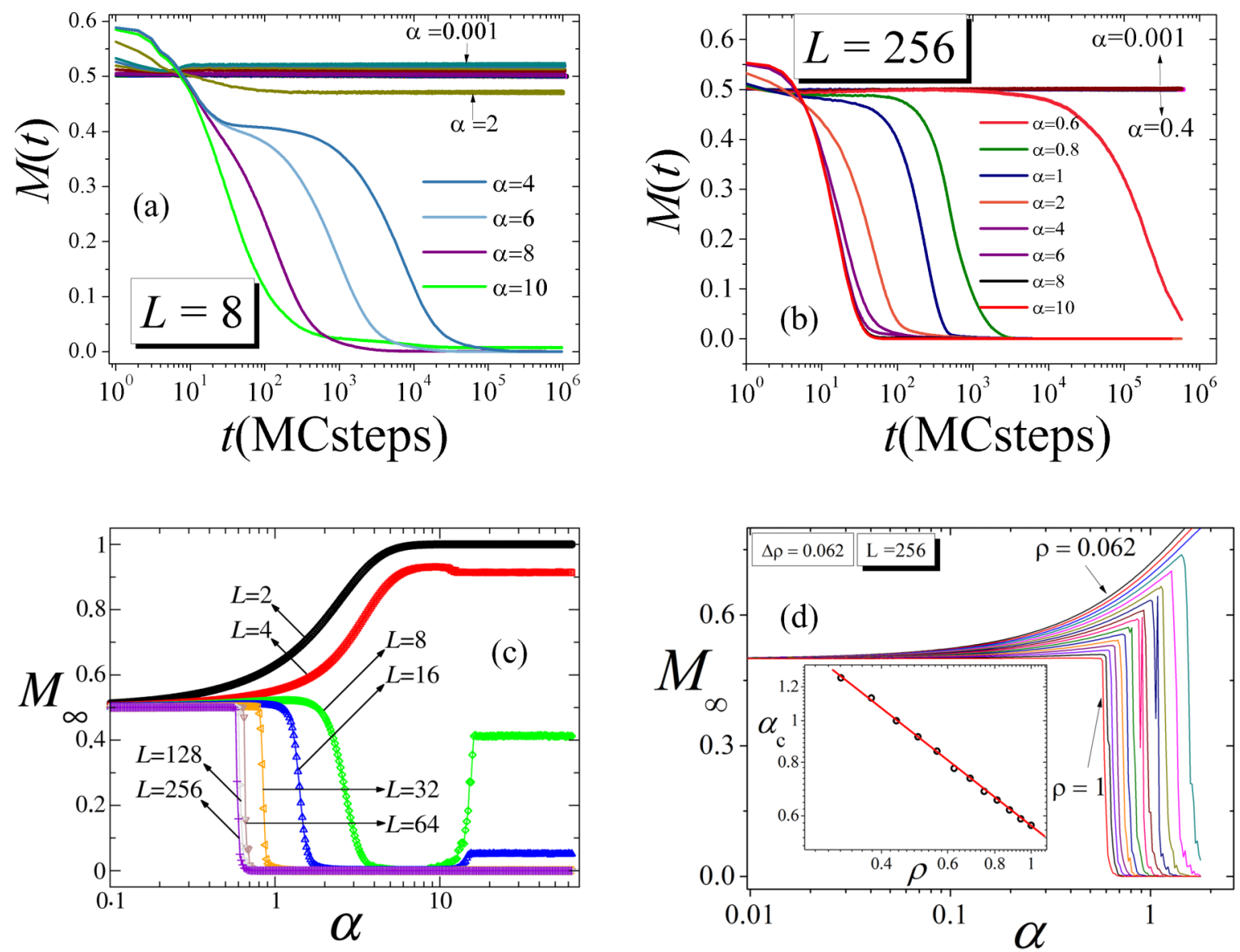

FIG. 4. (a) Time evolution of mobility for $L=8$. (b) Time evolution of mobility for $L=256$. (c) Finite size scaling of stationary mobility as function of $\alpha$. (d) Stationary mobility as function of $\alpha$ for fixed densities. An abrupt transition from mobile state to clogging state for $\alpha=\alpha_{c}$, can be observed which depends on density. The inset plot (in log-log scale) shows the dependence of $\alpha_{c}$ as function of $\rho$.

and in another simulation, we change $\sigma_{\max }$ keeping constant the occupation. And two surprising results are observed.

Figure 5 shows two distinct situations where we variate $\sigma_{\max }$. First keeping the density constant at $\rho=1$ and in a second case, keeping the occupation constant at $o=1$. In
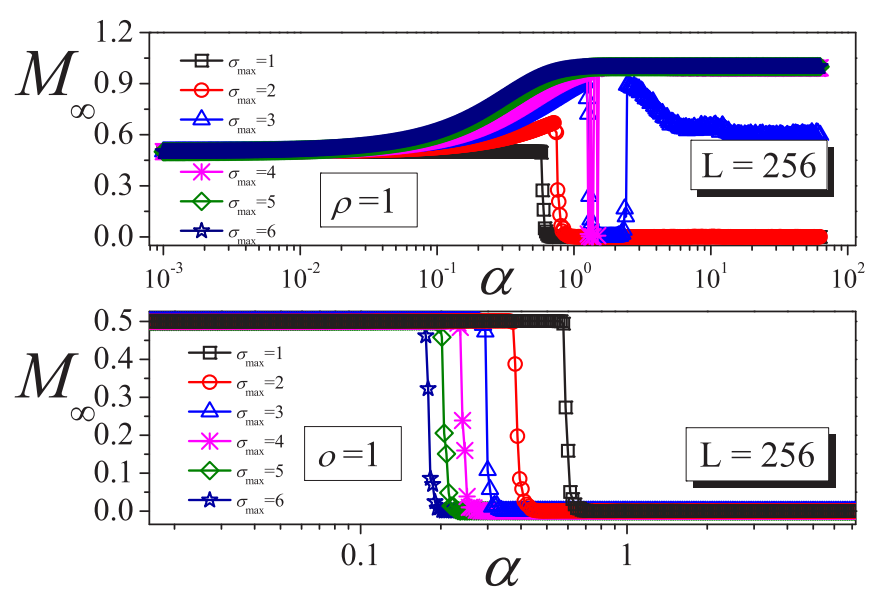

FIG. 5. Stationary mobility in two situations: $\rho=1$ and $o=1$, for different values of maximal ocupation: $\sigma_{\max }=1,2, \ldots, 6$. the first case we can observe a recovering of mobility for $\sigma_{\max }=3$ and $\sigma_{\max }=4$ and for $\sigma_{\max }>4$ the system does not present a formation of condensates for no value of $\alpha$, i.e., we can transit from a situation of objects interacting randomly with the environment to a situation where the objects strongly interact among them without the influence of the environment, and no bottleneck is observed since the object size in relation to cell size allows such situation. However, in the anomalous cases $\sigma_{\max }=3$ and $\sigma_{\max }=4$, the clogging occurs as in the case $\sigma_{\max }=1$, but the mobility is recovered (anomalously) for higher values of $\alpha$, given that the absence of randomness from the environment combined with intermediate relation between the object size and cell size. Here, differently from anomalies for very small systems, we believe that such point could also be due to an artefact of the synchronous MC simulations, which should be fixed by performing asynchronous MC simulations which deserves part of our future attention.

However, by keeping $o=1$, we did not wait a change in the critical value $\alpha_{c}$, since we enlarge $\sigma_{\max }$ as well as we enlarge the number of objects to maintain $o$ constant. This nonlinear response is characteristic of the Fermi-Dirac distribution of the cells for the transition probability. Such effects deserve more future investigation. 


\section{SUMMARIES, CONCLUSIONS, AND DISCUSSIONS}

In this work we show a different model that works with a parameter $\alpha$ that controls the randomness of the system by changing how the objects interact with the environment and among themselves. We observe a transition between a mobile phase and clogged phase in a $\alpha_{c}$ which depends on occupation of objects.

It is important to mention that such phenomena have some similarities with analogous models. For example, Helbing et al. [14], considering a simple model of particles driven in opposite directions and interacting via a repulsive potential, have found a transition to a crystallized state from a fluid state by increasing the amount of fluctuations of the system. The existence of condensates, here observed as function of $\alpha$, also was analyzed in another interesting and beautiful way by Majundar et al. [15], considering the shape of equilibrium mass distribution that change as the global mass density change. However, the authors have studied such a model only considering a single species, which suggests that such a model can be changed to cover our results.

By concluding, we believe that our model deserves more future explorations to better understand the mobile-clogging transition in such systems of counterflowing streams of particles via both analytical and computational methods.

\section{ACKNOWLEDGMENTS}

R. da Silva and E. V. Stock were financially supported by CNPq under Grants No. 311236/2018-9, No. 424052/20180 , and No. 154822/2016-7. This work was partly developed using the resources of Cluster Ada, IF-UFRGS. Finally, the authors are very grateful to Prof. Sandra Prado for the helpful suggestions to this manuscript.
[1] S. Machlup, J. Appl. Phys. 35, 341 (1954).

[2] M. J. Kirton and M. J. Uren, Adv. Phys. 38, 367 (1989).

[3] R. da Silva, L. C. Lamb, and G. I. Wirth, Philos. T. Roy. Soc. A 369, 307 (2011); R. da Silva, L. Brusamarello, and G. Wirth, Physica A 389, 2687 (2010); R. da Silva and G. I. Wirth, J. Stat. Mech. (2010) P04025; R. da Silva, G. I. Wirth, and L. Brusamarello, Int. J. Mod. Phys. B 24, 5885 (2010); J. Stat. Mech. (2008) P10015.

[4] R. da Silva, L. C. Lamb, Eder C. Lima, and J. Dupont, Physica A 391, 1 (2012).

[5] C. L. N. Oliveira, A. P. Vieira, D. Helbing, J. S. Andrade, Jr., and H. J. Herrmann, Phys. Rev. X 6, 011003 (2016).

[6] T. Vissers, A. Wysocki, M. Rex, H. Lowen, C. P. Royall, A. Imhof, and A. van Blaaderen, Soft Matter 7, 2352 (2011).

[7] T. Vissers, A. van Blaaderen, and A. Imhof, Phys. Rev. Lett. 106, 228303 (2011).
[8] E. V. Stock, R. da Silva, and H. A. Fernandes, Phys. Rev. E 96, 012155 (2017).

[9] R. da Silva, A. Hentz, and A. Alves, Physica A 437, 139 (2015).

[10] B. Schmittmann, N. Hwang, and R. K. P. Zia, Europhys. Lett. 19, 19 (1992).

[11] W. Feller, An Introduction to Probability Theory and Its Applications (Wiley, New York, 1966).

[12] J. Wei, H. Zhang, Y. Guo, and M. Gu, Phys. Lett. A 379, 1081 (2015).

[13] F. Alonso-Marroquin, J. Busch, C. Chiew, C. Lozano, and A. Ramırez-Gomez, Phys. Rev. E 90, 063305 (2014).

[14] D. Helbing, I. J. Farkas, and T. Vicsek, Phys. Rev. Lett. 84, 1240 (2000).

[15] S. N. Majumdar, M. R. Evans, and R. K. P. Zia, Phys. Rev. Lett. 94, 180601 (2005). 